\title{
Measurement of Pulsed Current-Voltage Characteristics of AlGaN/GaN HEMTs from Room Temperature to $15 \mathrm{~K}$
}

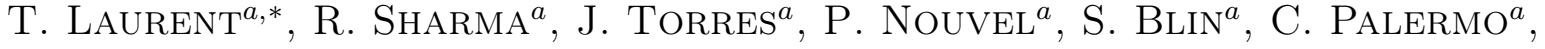 \\ L. VArAni ${ }^{a}$, Y. Cordier ${ }^{b}$, M. Chmielowska ${ }^{b}$, J.-P. FAURIE $^{c}$, And B. Beaumont $^{c}$ \\ ${ }^{a}$ Institut d'Electronique du Sud, UMR 5214 — CNRS/Université Montepellier 2 \\ Pl. E. Bataillon, 34095 Montpellier, France \\ ${ }^{b}$ Centre de Recherche sur l'Hétéro-Epitaxie et ses Applications, UPR 10 - CNRS \\ R. Bernard Grégory, 06560 Valbonne, France \\ ${ }^{c}$ LUMILOG, 2720, Ch. Saint Bernard Les Moulins I, 06220 Vallauris, France
}

\begin{abstract}
We report measurements of the pulsed and dc current-voltage characteristics of AlGaN/GaN high-electron-mobility transistors as functions of geometry, temperature (from 300 down to $15 \mathrm{~K}$ ), and operating conditions. An increase in the drain current with shortening of the pulse width from $1 \mu \mathrm{s}$ to $400 \mathrm{~ns}$ is found to be significant at room temperature whilst this behavior is inverted or even removed at 77 and $15 \mathrm{~K}$ temperatures.
\end{abstract}

PACS: 72.80.Ey, 73.21.Fg, 73.40.-c

\section{Introduction}

In recent years, the AlGaN/GaN HEMTs have become promising candidates because of their applications as high power and high temperature microwave devices [1]. In particular, the AlGaN/GaN HEMTs show high transconductance, high cut-off frequencies and good thermal management [2]. For example, the dispersion effects [3], the kink effect at 300 and $100 \mathrm{~K}$ temperatures [4], the self-heating effects [5], are among recent reports in the literature [6]. However, few investigations exist in the literature dealing with pulsed measurements especially at low temperature. In this letter, we report the pulsed and continuous $I-V$ characteristics of $\mathrm{AlGaN} / \mathrm{GaN}$ HEMTs as functions of geometry, temperatures (from 300 down to $15 \mathrm{~K}$ ) and operating conditions.

\section{Experimental setup}

The experimental setup used for the dc $I-V$ measurements consists of a dual-channel system source-meter, and the device under test (DUT) installed inside a cryostat for varying the temperature from 300 to 77 and $15 \mathrm{~K}$. The voltage sweep is performed for $5 \mathrm{~ms}$ between two voltage points of the dc $I-V$ curves. The experimental setup shown in Fig. 1 is used for the pulsed $I-V$ measurements where the source-meter is replaced by a function

* corresponding author; e-mail:

thibault. laurent@ies.univ-montp2.fr generator producing a voltage square pulse $\left(V_{\text {applied }}\right.$ in the figure) with a repetition rate of $100 \mu \mathrm{s}$. An oscilloscope is introduced to track the temporal profile of short (400 ns) and long $(1 \mu \mathrm{s})$ pulses. The drain current is calculated from the voltage drop across the $50 \Omega$ resistance of the function generator.

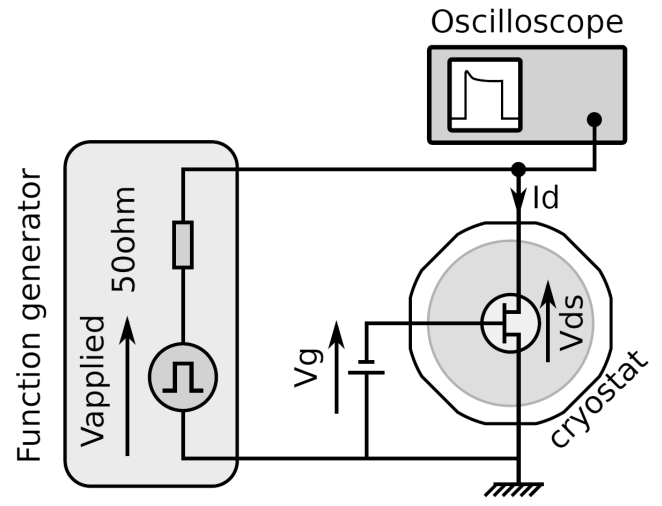

Fig. 1. Block diagram of the experimental setup used for pulsed $I-V$ measurements at 300,77 and $15 \mathrm{~K}$ temperatures.

The sample is cooled down at $77 \mathrm{~K}$ by using a flow of liquid nitrogen, then cooled down to $15 \mathrm{~K}$ by using a helium compressor.

As far as the fabrication process of the GaN HEMTs is concerned, the different layers consist of a sapphire substrate, a GaN iron doped template, a GaN $1 \mu \mathrm{m}$ thick buffer, a $1 \mathrm{~nm} \mathrm{AlN} / 21 \mathrm{~nm} \mathrm{Al}{ }_{0.28} \mathrm{Ga}_{0.72} \mathrm{~N} / 3 \mathrm{~nm} \mathrm{GaN} \mathrm{ac-}$ 
tive layer covered with $\mathrm{Ti} / \mathrm{Al}$ for ohmic and $\mathrm{Ni} / \mathrm{Au}$ for the Schottky contacts. The layers are grown and processed as described in [7]. The devices are not passivated. An ohmic contact resistance from 1 to $3 \mathrm{~W} \mathrm{~mm}$ is obtained depending on the sample. Capacitance voltage and the Hall measurements attest the presence of a two-dimensional electron gas with a carrier concentration of $10^{13} \mathrm{~cm}^{-2}$ and a mobility of $2000 \mathrm{~cm}^{2} /(\mathrm{V} \mathrm{s})$.

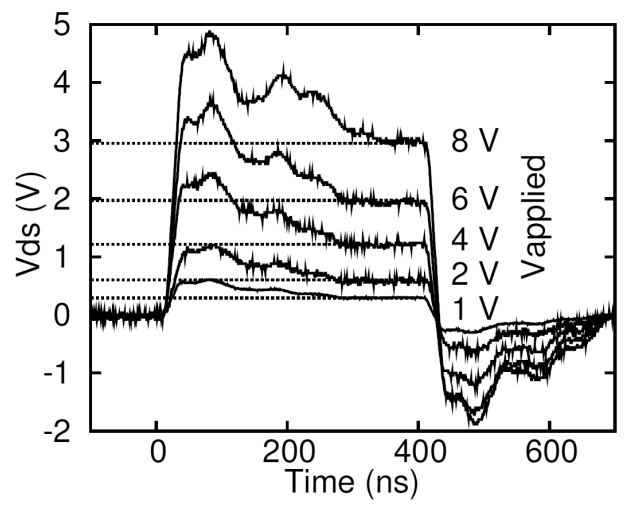

Fig. 2. Temporal evolution of drain-source voltage for varying the voltage from $1 \mathrm{~V}$ to $8 \mathrm{~V}$ at a fixed gate voltage of $-1 \mathrm{~V}$.

The temporal profiles of the pulses are found to be fairly stable during the measurements. Nonetheless, the situation shown by Fig. 2, for a short pulse (400 ns) at low temperature $(15 \mathrm{~K})$, is the worst and limiting case in terms of stability and accuracy of the recorded pulses. The shape of the pulse is stable and nearly a square-pulse for $1 \mathrm{~V}$ whilst the shape is considerably altered on increasing the drain voltage from 1 to $8 \mathrm{~V}$. The transient behavior at the beginning of the pulses may arise from the impedance of wires, connections, parasitic reactance and their resonant response to the pulses excitation. Since the voltage is stable in the final part of the pulses, the device voltage and current values are extracted by performing a time average of this portion.

\section{Results and discussion}

The $I-V$ characteristics of the GaN HEMTs having geometry, $L_{\mathrm{g}}=3 \mu \mathrm{m}, L_{\mathrm{ds}}=15 \mu \mathrm{m}$, are shown in Fig. 3a for an applied gate voltage of $-3 \mathrm{~V}$. We start the discussion of the experimental results by analyzing the HEMT behavior at room temperature. The lower values of the drain current in dc regime is attributed to the self-heating effect that is consistent with recent reports [3, 4]. The dc curve shows relatively lower values of the drain current $I_{\mathrm{d}}$ while its value is increased on shortening the pulse-widths from $1 \mu \mathrm{s}$ down to $400 \mathrm{~ns}$. The pulsed $I-V$ current values overpass the dc ones because the heating effects are reduced in the case of the pulsed measurements, and hence the drain current is enhanced. On the other hand, the heating effect dominates in the case of the dc
$I-V$ measurements thus reducing the current. A similar kind of enhancement in the drain current on shortening the pulse width is obtained for longer geometries $\left(L_{\mathrm{g}}=4 \mu \mathrm{m}, L_{\mathrm{ds}}=16 \mu \mathrm{m}\right)$ (Fig. 3b) except for the fact that the difference of the drain current values is sharply reduced for $1 \mu \mathrm{s}$ and $400 \mathrm{~ns}$ pulse voltages.

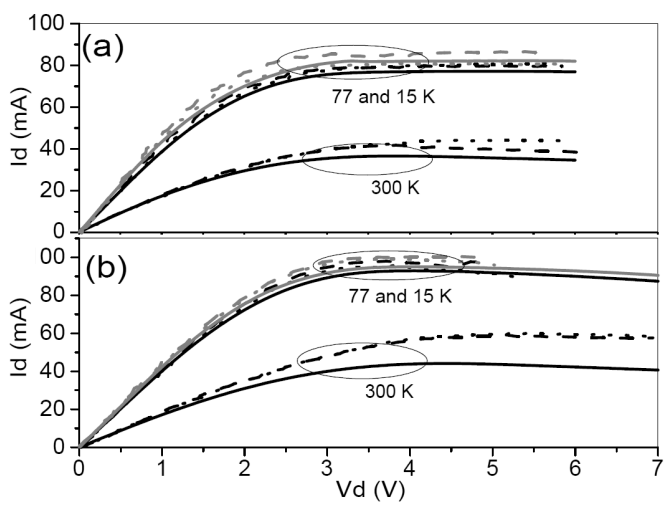

Fig. 3. Current-voltage characteristics of the GaN HEMTs with geometries of $L_{\mathrm{g}}=3 \mu \mathrm{m}$ and $L_{\mathrm{ds}}=15 \mu \mathrm{m}$ (a) with a gate voltage $V_{\mathrm{g}}=-3 \mathrm{~V}$, and $L_{\mathrm{g}}=4 \mu \mathrm{m}$ and $L_{\mathrm{ds}}=16 \mu \mathrm{m}$ (b) with a gate voltage $V_{\mathrm{g}}=-1 \mathrm{~V}$ at 300 (black curves), 77 (black curves) and $15 \mathrm{~K}$ (gray curves) temperatures. The continuous measurements are shown by solid line curves whilst the pulsed measurements, with varying pulse widths of $1 \mu$ s and $400 \mathrm{~ns}$, are indicated by dashed and dotted line curves, respectively.

Next, we investigate the dc and pulsed $I-V$ characteristics of the device under study at low temperatures. In the case of relatively small geometries, the drain current is enhanced by a considerable value on cooling the sample down to $77 \mathrm{~K}$. This means that the dynamics of the charge carriers is significantly affected by the cooling effect between 300 and $77 \mathrm{~K}$ due to the reduced phonon interactions. Between 77 and $15 \mathrm{~K}$, the drain current has practically attained its maximum value and only a small increase is measured (refer to topmost parts of Fig. 3a). The effect of changing the pulse width on the drain current at 77 and $15 \mathrm{~K}$ is removed and inverted, respectively, compared to $300 \mathrm{~K}$. For smaller geometries at $77 \mathrm{~K}$, the drain current remains the same for $1 \mu$ s and $400 \mathrm{~ns}$ pulse voltages but the $I_{\mathrm{d}}$ still has lower value. A similar kind of behavior is also seen at $15 \mathrm{~K}$ except for the fact that the drain current has relatively higher values at $15 \mathrm{~K}$ compared to $77 \mathrm{~K}$. In Ref. [3], it was shown that the drain-source current at $100 \mathrm{~K}$ for $V_{\mathrm{g}}=0 \mathrm{~V}$ is almost the same for the dc as well as for the pulsed measurements. The drain current was also found to have a lower value for the pulsed measurements compared to the dc ones for $V_{\mathrm{g}}=-1$ to $-3 \mathrm{~V}$. However, in our measurements, the effect of altering the pulse voltages on the drain current is dependent on the geometry. We stress that probably the effect of current enhancement by decreasing the pulse width was not evidenced in the results of Ref. [3] at high $V_{\mathrm{g}}$ due to the fact that the temperature was decreased only up to $100 \mathrm{~K}$. Recalling the fact that the self-heat 
could be easily dissipated in the smaller devices, the drain current increases on reducing the pulse voltage from $1 \mu \mathrm{s}$ to $400 \mathrm{~ns}$ (Fig. 3a). One can also see that the drain current at $15 \mathrm{~K}$ is nearly the same for $400 \mathrm{~ns}$ and the dc measurements (Fig. 3a) which indicate that the effect of altering the pulse voltage is removed in this case. On the other hand, in the case of relatively longer geometry at $77 \mathrm{~K}$, the drain current has nearly the same values for the dc as well as $1 \mu \mathrm{s}$ and $400 \mathrm{~ns}$ pulse voltages. There is also a negative slope at higher $V_{\mathrm{d}}$. However, the value of drain current is lower in the case of $400 \mathrm{~ns}$ pulse voltage compared to $1 \mu \mathrm{s}$ which indicates that the effect of the variation of the pulse-width voltage is inverted in this case.

It is interesting to note that the above discussed inversion behaviour and even its removal (refer to $400 \mathrm{~ns}$ and the dc results) suggests that the traps at 77 and $15 \mathrm{~K}$ may play a different role in the motion of the carriers. However, in the driving circuit of Fig. 1, the voltage step appearing between drain and source, $V_{\mathrm{ds}}$, not only affects the drain of the HEMT, but also its gate at the beginning of the pulse. Indeed, it should be pointed out that it is very difficult to get rid of the parasitic reactance present in the circuit due to the wiring of the samples in the cryostat and for this reason, we performed a time average on the end part of the pulses. We repeated these experiments on several samples and found similar results. Hence, we believe that the observed inversion behaviour is due to the variation of the pulse voltages, although it is not possible to get rid of the parasitic effects.

It is also noteworthy that we have not noticed any pronounced kink for all studied geometries at both room and cryogenic temperatures. As the presence of kinks is often attributed to impact ionization and trapping effects, this indicates the good quality of the studied sample material. It should be pointed out that the effect is dominant in the case of surface degradation of the sample, and it depends on the sample quality. Consequently, the observation of the kink may be due to the different fabrication processes of HEMTs, and geometries [3] compared to those which we are using.

\section{Conclusion}

In summary, we have experimentally investigated dc and pulsed current-voltage characteristics of AlGaN/ GaN HEMTs as functions of geometry, temperature, and operating conditions. We emphasize that all reported results have been found to be stable and reproducible in time and on different samples. The increase in the drain current with shortening of the pulse width is found to be significant at room temperature whilst this behavior is not only inverted but also ceased at 77 and $15 \mathrm{~K}$.

\section{Acknowledgments}

The French National Research Agency (ANR) and the Lithuanian Science Council are sincerely acknowledged for funding the research work under contract AITHER no. ANR-07-BLAN-0321 and grant no. MIP$-87 / 2010$. This work was partially supported by GIS 053713 TeraLab Montpellier.

\section{References}

[1] J. Kuzmik, Semicond. Sci. Technol. 17, 540 (2002).

[2] V. Kumar, A. Kuliev, R. Schwindt, M. Muir, G. Simin, J. Yang, M. Khan, I. Adesida, Solid-State Electron. 47, 1577 (2003).

[3] C. Lin, W. Wang, P. Lin, C. Lin, Y. Chang, Y. Chan, IEEE Electron Dev. Lett. 26, 710 (2005).

[4] A. Parker, J. Rathmell, IEEE Trans. Microwave Theory Techn. 49, 2105 (2001).

[5] J. Her, K. Lee, S. Lee, J. Lee, J. Oh, M. Han, K. Seo, Jpn. J. Appl. Phys. 44, 2726 (2005).

[6] K. Horio, A. Nakajima, K. Itagaki, Semicond. Sci. Technol. 24 (2009), doi: 10.1088/0268-1242/24/8/ 085022 .

[7] Y. Cordier, M. Azize, N. Baron, S. Chenot, O. Tottereau, J. Massies, J. Cryst. Growth 309, 1 (2007). 